\title{
SYMMETRIC HOMOLOGY OVER RINGS CONTAINING THE RATIONALS
}

\author{
PATRICK J. FLEURY
}

\begin{abstract}
Let $R$ be a commutative ring with unit which contains the rational numbers. Let $A$ be a commutative $R$-algebra. In this paper we prove that the cotriple homology and cohomology modules of $R$ for the symmetric algebra cotriple are the same as Harrison's homology and cohomology modules.
\end{abstract}

In [1], Barr showed that Harrison's homology theory over a field of characteristic zero was the same as cotriple homology for the symmetric algebraic cotriple. In order to do this, he relied on the fact that, over a field, the symmetric algebra of a module is a polynomial algebra and, thus, is projective. However he did conjecture that his theorem was true even when the ground ring was not a field but merely was a ring containing the rational numbers. The purpose of this paper is to prove that conjecture. This is part of the author's dissertation at the University of Illinois. The author would like to express his apreciation to Professor Barr for all the advice and encouragement received.

1. Preliminaries. We refer to [1] for the definition of cotriple and cotriple homology. Let $k$ be a commutative ring, ${ }_{k} \boldsymbol{M}$ the category of left $k$-modules and ${ }_{k} \mathrm{Alg}$ the category of commutative $k$-algebras. There is a functor $U:{ }_{k} \mathrm{Alg} \rightarrow{ }_{k} M$ which assigns to each $k$-algebra its underlying $k$ module and to each algebra morphism its underlying module morphism. It is well known that this functor has a left adjoint $S:{ }_{k} \boldsymbol{M} \rightarrow{ }_{k}$ Alg called the symmetric algebra functor It can then be shown easily that the composition $S U$ together with natural transformations $\varepsilon: S U \rightarrow_{k} \mathrm{Alg}$ (multiplication) and $\delta: S U \rightarrow S U S U$ (front adjunction) is a cotriple which we shall now abbreviate to $S$.

Now suppose $A$ is a commutative $k$-algebra and let us consider the $k$-algebras, $A, S A, S^{2} A, \cdots$. We have a map $\varepsilon A: S A \rightarrow A$. This gives rise to two maps $S \varepsilon A, \varepsilon S A: S^{2} A \rightarrow S A$. In general, we have $n+1$ maps from $S^{n+1} A$ to $S^{n} A$ given by $S^{n-i} \varepsilon S^{i} A$ for $i$ ranging from zero to $n$. We also

Received by the editors November 30, 1972.

AMS (MOS) subject classifications (1970). Primary $18 \mathrm{H} 20$.

Key words and phrases. Harrison (co)-homology, cotriple (co)-homology, Koszul resolution. 
have maps from $S^{n+1} A$ to $S^{n+2} A$ given by $S^{n-i} \delta S^{i} A$. Huber has shown in [8] that $A, S A, S^{2} A, \cdots$ together with the maps defined above form an augmented simplicial object over the category of commutative $k$-algebras.

There is a unique map from $S^{n} A$ to $A$ which is arrived at by taking any composite of the $S^{n-i} \varepsilon S^{i} A$ 's. We now obtain a complex of $A$-modules by taking

$$
\cdots \rightarrow \text { Diff } S^{n} A \rightarrow \operatorname{Diff} S^{n-1} A \rightarrow \cdots \rightarrow \text { Diff } S A \rightarrow 0
$$

where the boundary maps are alternating sums of faces of the simplicial object and where Diff is defined as follows. If $A^{\prime}$ is a commutative $k$ algebra and $\varphi: A^{\prime} \rightarrow A$ is an algebra morphism we set Diff $A^{\prime}=A \otimes A^{\prime} / N$ where $N$ is that submodule of $A \otimes A^{\prime}$ generated by all elements of the form $a \otimes a_{1}^{\prime} \cdot a_{2}^{\prime}-a \varphi\left(a_{1}^{\prime}\right) \otimes a_{2}^{\prime}-a \varphi\left(a_{2}^{\prime}\right) \otimes a_{1}^{\prime}$. If $M$ is an $A$-module, it is easily seen that $\operatorname{Hom}_{A}\left(\operatorname{Diff} A^{\prime}, M\right) \cong \operatorname{Der}\left(A^{\prime}, M\right)$ where $\operatorname{Der}\left(A^{\prime}, M\right)$ is the abelian group of all derivations from $A^{\prime}$ to $M$.

We now define the symmetric homology of $A$ with coefficients in the $A$-module $M$ to be the homology of the complex

$\cdots \rightarrow$ Diff $S^{n} A \otimes_{A} M \rightarrow$ Diff $S^{n-1} A \otimes_{A} M \rightarrow \cdots \rightarrow$ Diff $S A \otimes_{A} M \rightarrow 0$

and we denote the $n$th homology module by $\operatorname{Symm}_{n}(A, M)$. Similarly, we define the symmetric cohomology of $A$ to be the homology of the complex

$$
0 \rightarrow \operatorname{Hom}_{A}(\text { Diff } S A, M) \rightarrow \operatorname{Hom}_{A}\left(\operatorname{Diff} S^{2} A, M\right) \rightarrow \cdots .
$$

We denote the $n$th symmetric cohomology module by $\operatorname{Symm}^{n}(A, M)$ and note that it is also the homology of the following complex

$$
0 \rightarrow \operatorname{Der}(S A, M) \rightarrow \operatorname{Der}\left(S^{2} A, M\right) \rightarrow \cdots .
$$

We now recall Harrison's homology theory. If $A$ is a commutative $k$ algebra, then we define the $n$th dimension of Hochschild's complex $C_{*} A$ to be $A \otimes A^{(n)}$ provided we take coefficients only in left (symmetric) $A$ modules (see [1]). We then define shuffle maps $s_{i, n-i}: A \otimes A^{(n)} \rightarrow A \otimes A^{(n)}$ inductively as follows.

$$
\begin{gathered}
s_{0, n}=s_{n, 0}=\mathrm{id}, \\
s_{i, n-i}\left(a_{0} \otimes a_{1} \otimes \cdots \otimes a_{n}\right) \\
=a_{0} \otimes a_{1} \otimes s_{i-1, n-i}\left(a_{2} \otimes \cdots \otimes a_{n}\right) \\
\quad+(-1)^{i} a_{0} \otimes a_{i+1} \otimes s_{i, n-i-1}\left(a_{1} \otimes \cdots \otimes \hat{a}_{i+1} \otimes \cdots \otimes a_{n}\right)
\end{gathered}
$$

where "^" signifies deletion. We recall from [1] that the sum of the images of the shuffles forms a subcomplex of $C_{*} A$ called $\mathrm{Sh}_{*} A$. We then set Harrison's complex, $\mathrm{Ch}_{*} A$, equal to the quotient complex $C_{*} A / \mathrm{Sh}_{*} A$. If 
$M$ is a left $A$-module, we set

$$
\begin{aligned}
& \operatorname{Harr}_{n}(A, M)=H_{n}\left(\mathrm{Ch}_{*} A \otimes_{A} M\right), \\
& \operatorname{Harr}^{n}(A, M)=H_{n}\left(\operatorname{Hom}_{A}\left(\mathrm{Ch}_{*} A, M\right)\right) .
\end{aligned}
$$

The main theorem of this paper is then

THEOREM 1.1. Let $k$ be any commutative ring containing the rational numbers. Let $A$ be any commutative $k$-algebra and $N$ any left $A$-module. Then

$$
\operatorname{Symm}_{n}(A, N) \cong \operatorname{Harr}_{n+1}(A, N), \quad \operatorname{Symm}^{n}(A, N) \cong \operatorname{Harr}^{n+1}(A, N) .
$$

2. The main lemma. Theorem 1.1 relies heavily on the following lemma and the resolution we use to prove it. From this point on, we shall always assume that $k$ is a commutative ring containing the rational numbers.

LEMMA 2.1. Let $M$ be a k-module and $N$ an SM-module. Then $\operatorname{Harr}_{n}(S M, N)=0=\operatorname{Harr}^{n}(S M, N)$ for $n>1$. Furthermore $\operatorname{Harr}_{1}(S M, N)=$ $\operatorname{Diff} S M \otimes_{S M} N$ and $\operatorname{Harr}^{1}(S M, N)=\operatorname{Der}(S M, N)$.

Proof. The second part of the lemma is a direct calculation which we omit. In order to prove the first part of the lemma, we must make use of the techniques of relative homological algebra. Since our interest is not in this subject as such, we refer the reader to Chapter 9 of [10] for an exposition of it.

With this in mind, we note that $\operatorname{Hoch}_{i}(S M, N)=\operatorname{Tor}_{i}^{(S M \otimes S M, k)}(S M, N)$ where the right-hand side stands for $(S M \otimes S M, k)$-relative homology theory. Similarly, we see that $\left.\operatorname{Hoch}^{i}(S M, N)=\operatorname{Ext}_{(S M \otimes S M, k)}\right)(S M, N)$. We now shall examine a resolution of $S M$ which will allow us to calculate these groups in more detail.

Let $M^{\#}$ be a $k$-module isomorphic to and distinct from $M$. Let the isomorphism send $m \varepsilon M$ to $m^{\#} \varepsilon M^{\#}$. Then $S M^{\#}$ is isomorphic to $S M$. Thus $\operatorname{Tor}_{i}^{(S M \otimes S M, k)}(S M, N) \cong \operatorname{Tor}_{i}^{(S M \otimes S M \#, k)}(S M, N)$ for any symmetric $S M$-bimodule, $N$, where the action of $S M^{\#}$ is defined via the isomorphism. Let $M^{\prime}$ be that submodule of $S M \otimes S M^{\#}$ generated by elements of the form $1 \otimes m^{\#}-m \otimes 1$. Then $M^{\prime}$ is isomorphic to $M$ so $S M \otimes S M^{\prime} \cong S M \otimes S M$. Then as an $S M \otimes S M^{\prime}$ module, $N$ is isomorphic to $N \otimes k$ where $S M^{\prime}$ acts on the ground ring $k$ via the usual augmentation map $S M^{\prime} \rightarrow k$. Thus we have $\operatorname{Tor}_{i}^{(S M \otimes S M, k)}(S M, N) \cong \operatorname{Tor}_{i}^{\left(S M \otimes S M^{\prime}, k\right)}(S M \otimes k, N \otimes k)$. We will now construct the Koszul complex of $\left(S M^{\prime}, k\right)$-relative free modules and use this complex to get a useful $\left(S M \otimes S M^{\prime}, k\right)$-relatively free allowable resolution of $S M \otimes k$.

For convenience, we shall use $M$ rather than $M^{\prime}$ for the time being and 
make adjustments later. Let $X_{n}$ be $S M \otimes \bigwedge_{n} M$ where $\bigwedge_{n} M$ stands for the exterior product of $M$ with itself $n$ times. We define a boundary operator $d_{n}: X_{n} \rightarrow X_{n-1}$ by

$$
\begin{aligned}
& d_{n}\left(m_{1} \cdot \cdots \cdot m_{r} \otimes m_{r+1} \wedge \cdots \wedge m_{r+n}\right) \\
& \quad=\sum_{i=1}^{n}(-1)^{i-1}\left(m_{1} \cdots \cdot m_{r} \cdot m_{r+i} \otimes m_{r+1} \wedge \cdots \wedge \hat{m}_{r+i} \wedge \cdots \wedge m_{r+n}\right)
\end{aligned}
$$

where $\hat{m}_{r+i}$ signifies the deletion of $m_{r+i}$. It is easy to see that $d_{n}$ is a well-defined $S M$-module homomorphism and that $d_{n-1} d_{n}=0$.

We must now show that this complex is $k$-split. To define a $k$-module morphism from $X_{n}$ to $X_{n+1}$ we need to define it on each $k$-summand of $S M \otimes \bigwedge_{n} M$. We set $t_{r, n}\left(m_{1} \cdots \cdots m_{r} \otimes m_{r+1} \wedge \cdots \wedge m_{r+n}\right)=0$ if $r=0$. If $r$ is not zero, then

$$
\begin{aligned}
t_{r, n}\left(m_{1} \cdots \cdots m_{r} \otimes m_{r+1} \wedge \cdots \wedge m_{r+n}\right) & \\
& =\frac{1}{r+n} \sum_{j=1}^{r}\left(m_{1} \cdots \hat{m}_{j} \cdots m_{r} \otimes m_{j} \wedge m_{r+1} \wedge \cdots \wedge m_{r+n}\right) .
\end{aligned}
$$

It is easy to see that $t_{r, n}$ is well defined, since if $U_{r, n}: M \times \cdots \times M \rightarrow$ $S_{r-1} M \otimes \bigwedge_{n+1} M$ is defined by the above formula, then it is $k$-linear, symmetric in the first $r$-variables and skew symmetric in the last $n$-variables. Thus $U_{r, n}$ has a unique factorization through $S_{r} M \otimes \bigwedge_{n} M$ which is $t_{r, n}$.

Let $t_{n}: X_{n} \rightarrow X_{n+1}$ be the map on the direct sum which has component $t_{r, n}$ on the $r$ th summand. Let $t_{-1}: k \rightarrow S M$ be front adjunction. It is then a straightforward although somewhat tedious calculation to show that the $t_{n}$ 's so defined form a $k$-contracting homotopy.

From the foregoing, we see that

$$
\cdots \rightarrow S M^{\prime} \otimes \bigwedge_{n} M^{\prime} \rightarrow S M^{\prime} \otimes \bigwedge_{n-1} M^{\prime} \rightarrow \cdots \rightarrow S M^{\prime} \otimes \bigwedge_{0} M^{\prime} \rightarrow k \rightarrow 0
$$

is an $\left(S M^{\prime}, k\right)$-relatively free allowable resolution of $k$. Thus

$$
\begin{aligned}
\cdots \rightarrow S M \otimes S M^{\prime} \otimes \bigwedge_{n} M^{\prime} \rightarrow & S M \otimes S M^{\prime} \otimes \bigwedge_{n-1} M^{\prime} \rightarrow \\
& \quad \cdots \rightarrow S M \otimes S M^{\prime} \otimes \bigwedge_{0} M^{\prime} \rightarrow S M \otimes k \rightarrow 0
\end{aligned}
$$

is an $\left(S M \otimes S M^{\prime}, k\right)$-relatively free allowable resolution of $S M \otimes k$. Thus $\operatorname{Tor}_{*}^{\left(S M \otimes S M^{\prime}, k\right)}(S M \otimes k, N \otimes k)$ is the homology of the complex which has $S M \otimes S M^{\prime} \otimes \bigwedge_{n} M^{\prime} \otimes S M \otimes S M^{\prime} N \otimes k$ in the $n$th dimension. But because the action of $M^{\prime}$ on $k$ is trivial and because we take the tensor product over $S M \otimes S M^{\prime}$ once, we see that the maps in this complex are all zero. Thus the $n$th homology module of this complex is just $\bigwedge_{n} M^{\prime} \otimes N \otimes k$. Similarly $\operatorname{Ext}_{\left(S M \otimes S M^{\prime}, k\right)}^{*}(S M \otimes k, N \otimes k)$ is the homology of the complex 
with $\mathrm{Hom}_{S, M \otimes S M^{\prime}}\left(S M \otimes S M^{\prime} \otimes \bigwedge_{n} M^{\prime}, N \otimes k\right)$ in the $n$th dimension. Again the differential turns out to be zero so the $n$th cohomology module is $\mathrm{Hom}_{k}\left(\bigwedge_{n} M^{\prime}, N \otimes k\right)$.

The shuffle product induces a map on Hochschild homology

$\operatorname{Hoch}_{p}(S M, S M) \otimes_{S M \otimes S M} \operatorname{Hoch}_{q}(S M, N) \rightarrow \operatorname{Hoch}_{p+q}(S M, N)$.

When we consider the Koszul complex, we find that the map induced is just exterior multiplication. Since this map is clearly surjective on generators, we conclude that every element of $\operatorname{Hoch}_{n}(S M, N)$ for $n \geqq 2$ is represented by a shuffle. In [1], Barr shows that Harrison's homology over a field of characteristic zero is a direct summand of Hochschild's homology and that the former consists of those homology classes which are not represented by shuffles. Barr's proof carries over to a ring containing the rationals, so we may conclude that $\operatorname{Harr}_{n}(S M, N)=0$ for $n \geqq 2$ since everything in $\operatorname{Hoch}_{n}(S M, N)$ is represented by a shuffle.

Similarly, there is a map

$\operatorname{Hoch}^{p+q}(S M, N) \rightarrow \operatorname{Hom}_{S M \otimes S M}\left(\operatorname{Hoch}_{p}(S M, S M), \operatorname{Hoch}^{q}(S M, N)\right)$.

If we use the Koszul complex, this map is given by the following. We pick $f \in \operatorname{Hom}_{k}\left(\bigwedge_{p+q} M^{\prime}, N \otimes k\right)$ and set

$$
\begin{aligned}
f\left(m_{1} \wedge\right. & \left.\cdots \wedge m_{p}\right)\left(m_{p+1} \cdot \cdots \cdot m_{p+r} \otimes 1\right)\left(\bar{m}_{1} \wedge \cdots \wedge \bar{m}_{q}\right) \\
& =\left(m_{p+1} \cdot \cdots \cdot m_{p+r} \otimes 1\right) f\left(m_{1} \wedge \cdots \wedge m_{p} \wedge \bar{m}_{1} \wedge \cdots \wedge \bar{m}_{q}\right) .
\end{aligned}
$$

This map is obviously injective so if $f$ disappears on exterior products, it must be zero. Thus if a cocycle disappears on shuffles it must be a coboundary. But Barr also shows that Harrison's cohomology classes are exactly those classes of the Hochschild Theory which are represented by cocycles which are zero on shuffles. Thus $\operatorname{Harr}^{n}(S M, N)=0$ for $n \geqq 2$. Thus Lemma 2.1 is proved.

3. The double complex. We now have all the tools needed to complete the proof of Theorem 1.1. Let us consider the double complex $E_{i, j}$ where $E_{i, j}=\mathrm{Ch}_{i+1}\left(S^{j+1} A\right) \otimes M$. There are two boundary maps $D_{i}^{\mathrm{I}}: E_{i, j} \rightarrow E_{i-1, j}$ which is the restriction of the Hochschild boundary and the second is $D_{j}^{I \mathrm{I}}: E_{i, j} \rightarrow E_{i, j-1}$ which is the cotriple boundary map for the cotriple $S$.

There is a map of $k$-modules $\psi A: A \rightarrow S A$ by front adjunction. This gives rise to a contracting homotopy in the complex

$$
\cdots \rightarrow S^{3} A \otimes M \rightarrow S^{2} A \otimes M \rightarrow S A \otimes M \rightarrow A \otimes M \rightarrow 0 .
$$

Thus the $n$-fold tensor product of the homotopy gives rise to a contracting homotopy in

$$
\cdots \rightarrow \mathrm{Ch}_{n}\left(S^{2} A\right) \otimes M \rightarrow \mathrm{Ch}_{n}(S A) \otimes M \rightarrow \mathrm{Ch}(A) \otimes M \rightarrow 0 .
$$


Thus, $H_{\mathrm{II}}(E)$ is simply the complex $\mathrm{Ch}_{*} A \otimes_{A} M$, so $H_{\mathrm{I}} H_{\mathrm{II}}(E)$ is $\operatorname{Harr}_{*}(A, M)$. On the other hand, we have shown that $H_{\mathrm{I}, j}(E)$ is Diff $S^{j+1} A \otimes_{A} M$ concentrated in bidegree $(j, 0)$. Thus $H_{\mathrm{I}} H_{\mathrm{II}}(E)$ is $\operatorname{Symm}_{*}(A, M)$ and by Theorem 6.1, p. 342 of $[10]$, we are done. The proof for cohomology is similar.

\section{REFERENCES}

1. M. Barr, Harrison homology, Hochschild homology and triples, J. Algebra 8 (1968), no. 3, 314-323. MR 36 \#3851.

2. M. Barr and J. Beck, Homology and standard construction, Seminar on Triples and Categorical Homology Theory (ETH, Zürich, 1966/67), Springer, Berlin, 1969, pp. 245-335. MR 41 \#3562.

3. J. Beck, Triples, algebras and cohomology, Dissertation, Columbia University, New York, 1967.

4. P. Fleury, Aspects of Harrison's homology theory, Dissertation, University of Illinois, Urbana, Illinois, 1970.

5. - Splittings of Hochschild's complex for commutative algebras, Proc. Amer. Math. Soc. 30 (1971), 405-411.

6. R. Godement, Topologie algébrique et théorie des faisceaux, Actualités Sci. Indust., no. 1252 = Publ. Math. Univ. Strasbourg, no. 13, Hermann, Paris, 1958. MR 21 \#1583.

7. D. Harrison, Commutative algebras and cohomology, Trans. Amer. Math. Soc. 104 (1962), 191-204. MR 26 \#176.

8. P. J. Huber, Standard constructions in abelian catagories, Math. Ann. 146 (1962), 321-325. MR 27 \#188.

9. J. Lambek, Lectures on rings and modules, Blaisdell, Waltham, Mass., 1966. MR 34 \#5857.

10. S. Mac Lane, Homology, Die Grundlehren der math. Wissenschaften, Band 114, Academic Press, New York; Springer-Verlag, Berlin, 1963. MR 28 \#122.

11. B. Mitchell, Theory of categories, Pure and Appl. Math., vol. 17, Academic Press, New York, 1965. MR 34 \#2647.

Department of Mathematics, State University College of Arts and Science, Plattsburgh, New York 12901 